\title{
Índices de vegetação simulados de diferentes sensores na estimativa das variáveis biofísicas do feijoeiro
}

\author{
Priscylla Ferraz Câmara Monteiro(1), Rubens Angulo Filho(2), \\ Alexandre Cândido Xavier(3) e Rodrigo Otávio Câmara Monteiro(4)
}

\begin{abstract}
(1)Fundação Estadual de Pesquisa Agropecuária, BR-285, Km 126, Caixa Postal 20, CEP 95200-970 Vacaria, RS. E-mail: priscylla-monteiro@fepagro.rs.gov.br (2)Universidade de São Paulo, Escola Superior de Agricultura Luiz de Queiroz, Departamento de Engenharia de Biossistemas, Avenida Pádua Dias, no 11, Caixa Postal 09, CEP 13418-900 Piracicaba, SP. E-mail: ruangulo@usp.br (3)Universidade Federal do Espírito Santo, Departamento de Engenharia Rural, Alto Universitário, s/no, Caixa Postal 16, CEP 29500-000 Alegre, ES. E-mail: alexandre.candido.xavier.ufes@gmail.com ${ }^{(4)}$ Instituto Federal de Educação, Ciência e Tecnologia, Departamento de Pesquisa e Inovação, Avenida Osvaldo Aranha, no 540, CEP 95700-000 Bento Gonçalves, RS. E-mail: rodrigo.monteiro@bento.ifrs.edu.br
\end{abstract}

Resumo - O objetivo deste trabalho foi avaliar os índices de vegetação e bandas do vermelho e do infravermelho próximo, gerados a partir dos sensores HRVIR, ETM+ e Modis, nas estimativas de índice de área foliar e produtividade da cultura do feijoeiro. O experimento foi realizado em blocos ao acaso, com parcelas subdivididas, com quatro lâminas de irrigação $(179,5,256,5,357,5$ e 406,2 mm), três doses de $\mathrm{N}(0,0,80,0$ e 160,0 kg ha-1) e quatro repetições. As medidas de reflectância foram obtidas com o Spetron SE-590, no estádio R6 da cultura, nas 48 parcelas. Foram testados: a razão simples, o índice de vegetação por diferença normalizada, índice de vegetação ajustado ao solo e índice de vegetação realçado. Os índices de vegetação foram eficientes na estimativa do índice de área foliar (IAF) e da produtividade da cultura do feijoeiro. Os índices de vegetação e a banda do infravermelho apresentam o mesmo potencial na estimativa do IAF, quando se considera a resolução espectral dos sensores Modis, ETM+ e HRVIR.

Termos de indexação: Phaseolus vulgaris, agricultura, reflectância espectral, sensoriamento remoto.

\section{Vegetation indices simulated from different sensors for the estimation of biophysical variables of common bean}

\begin{abstract}
The objective of this work was to evaluate the vegetation indices and red and infrared bands of the HRVIR, ETM+ and Modis sensors, in the estimations of leaf area index and grain yield of common bean. The experiment was carried out in a randomized block design, with split plots and four irrigation levels (179.5, 256.5, 357.5, and $406.2 \mathrm{~mm})$, three $\mathrm{N}$ rates $\left(0.0,80.0\right.$ and $\left.160.0 \mathrm{~kg} \mathrm{ha}^{-1}\right)$, and four replicates. The reflectance measurements were obtained through the Spectron SE-590, at R6 stage, on 48 plots. Simple ratio, normalized difference vegetation index, soil-adjusted vegetation index, and enhanced vegetation index were tested. The vegetation indices were efficient to estimate the leaf area index (LAI) and grain yield. The vegetation indices and infrared band show the same potential to estimate LAI, when considering the spectral resolution of Modis, ETM+ and HRVIR.
\end{abstract}

Index terms: Phaseolus vulgaris, agriculture, spectral reflectance, remote sensing.

\section{Introdução}

O feijão é uma das principais fontes de alimentação no mundo. Segundo a Food and Agriculture Organization of the United Nations (2013), o Brasil foi, em 2011, o terceiro maior produtor mundial de feijão, atrás apenas da Índia e Myanmar. No Brasil, as estimativas de produtividade agrícola são realizadas pelo IBGE (Instituto Brasileiro de Geografia e Estatística), com base em questionários, o que dificulta a análise da acurácia dos dados envolvidos. Assim, o sensoriamento remoto passa a ser uma ferramenta importante para a identificação das áreas ocupadas pelas culturas (Sanches et al., 2005), para a discriminação de variedades agrícolas (Galvão et al., 2009) e para a estimativa de parâmetros biofísicos utilizados em modelos de produtividade agrícola, como índice de área foliar (IAF) (Doraiswamy et al., 2004).

A relação entre o sensoriamento remoto e os parâmetros biofísicos pode ser feita por meio das bandas simples e, também, pela aplicação de índices de vegetação. Os índices de vegetação de banda larga são frequentemente utilizados para estimar parâmetros de vegetação, e a base física desses índices é atribuída 
à alta absorção da radiação pela clorofila e ao seu espalhamento pelas folhas, nas regiões espectrais do vermelho e do infravermelho próximo, respectivamente (Gates et al., 1965). Dentre os índices de vegetação usualmente utilizados, destacam-se: a razão simples (simple ratio - SR) (Gates et al., 1965); e o índice de vegetação por diferença normalizada (normalized difference vegetation index - NDVI) (Rouse et al., 1974), que é amplamente utilizado na estimativa de biomassa, do IAF e da produtividade (Asrar et al., 1984; Xavier \& Vettorazzi, 2004). Para minimizar o efeito das variações de brilho do solo, Huete (1988) desenvolveu o índice de vegetação ajustado ao solo (soil-ajusted vegetation index - Savi), em que se utilizam as mesmas bandas do NDVI, porém com um deslocamento dos eixos espectrais. Com o advento do sensor moderate resolution imaging spectroradiometer (Modis), houve o surgimento de um novo índice de vegetação, o índice de vegetação realçado (enhanced vegetation index - EVI), sensível à variação da estrutura do dossel, inclusive o IAF, a fisionomia da planta e a arquitetura do dossel (Huete et al., 1997).

Os índices de vegetação obtidos de imagens de satélites de média resolução espacial, como as do ETM+/Landsat 7 e HRVIR/Spot 4, podem ser utilizados para estimar a produtividade de culturas agrícolas. Entretanto, em razão da baixa resolução temporal (baixa disponibilidade de imagens no período de uma safra agrícola) e da alta probabilidade de incidência de nuvens durante o período de desenvolvimento das culturas, é viável a utilização do sensor Modis, a bordo dos satélites Terra e Aqua, com resolução temporal diária que possibilita um aumento considerável da disponibilidade de imagens livres de nuvens.

O objetivo deste trabalho foi o de avaliar os índices de vegetação SR, NDVI, Savi e EVI e bandas do vermelho e do infravermelho próximo, gerados a partir dos sensores HRVIR, ETM+ e Modis, nas estimativas de índice de área foliar e produtividade da cultura do feijoeiro.

\section{Material e Métodos}

O experimento foi realizado em condições de campo, de setembro a dezembro de 2007, em área experimental de agricultura irrigada, no Município de Piracicaba, SP, a $22^{\circ} 42^{\prime} 30^{\prime \prime} \mathrm{S}, 4^{\circ} 38^{\prime} 39^{\prime \prime} \mathrm{W}$, à altitude média de $547 \mathrm{~m}$.
Segundo a classificação de Köppen-Geiger, o clima da região é do tipo Cwa, mesotérmico (subtropical úmido), com estação chuvosa no verão e estiagem no inverno, com precipitação pluvial média anual de $1.247 \mathrm{~mm}$, temperatura média do ar de $21,6^{\circ} \mathrm{C}$ e umidade relativa do ar média de $74 \%$.

A área experimental tem solo classificado como Nitossolo Vermelho eutrófico, de horizonte Amoderado, profundo e permeável, com textura argilosa em todo o perfil, com um incremento de argila no horizonte B (Santos et al., 2006). A área foi preparada com uma aração e uma gradagem, nos dois dias antecedentes à semeadura.

Foi utilizada a cultivar de feijão Pérola. A semeadura foi realizada manualmente, no dia 04 de setembro de 2007, em sulcos de $0,05 \mathrm{~m}$ de profundidade. O desbaste foi realizado 15 dias após a semeadura, tendo-se deixado 14 plantas por metro linear. $\mathrm{O}$ espaçamento utilizado foi de $0,50 \mathrm{~m}$ entre fileiras de semeadura, no total de sete linhas por parcela. Cada parcela media 3,2 $\mathrm{m}$ de comprimento e 2,2 $\mathrm{m}$ de largura.

O experimento foi realizado em blocos ao acaso, com parcelas subdivididas e quatro repetições. Os tratamentos consistiram de níveis de irrigação $(179,5,256,5,357,5$ e 406,2 $\mathrm{mm}$ de água), nas parcelas, e doses de nitrogênio $\left(0,0,80,0\right.$ e $160,0 \mathrm{~kg} \mathrm{ha}^{-1}$ de $\left.\mathrm{N}\right)$, nas subparcelas, no total de 12 tratamentos. Quanto à adubação, um terço da dose total de $\mathrm{N}$ (sulfato de amônio) foi aplicado durante a semeadura, e outras duas aplicações foram feitas durante os estádios V2 - aos sete dias após a emergência (DAE), e V4 - aos 27 DAE, conforme recomendação de Soratto et al. (2004). As adubações de K e P foram de 90 e $100 \mathrm{~kg} \mathrm{ha}^{-1}$, respectivamente. A irrigação foi realizada por aspersão, conforme o sistema de aspersão em linha, (Hanks et al., 1976).

As medições do IAF foram realizadas por método não destrutivo, com uso do equipamento "Plant Canopy analyzer" LAI-2000, (LI-COR Biosciences, Lincoln, Nebraska-EUA). Para estimar o IAF, as medidas de radiação difusa, acima e abaixo do dossel do feijão, foram coletadas no início da manhã em 01/11/2007, em todas as parcelas, no estádio fenológico R6 (floração), conforme Gepts \& Fernandéz (1982). O estádio R6 foi caracterizado como o melhor estádio da cultura para estimar as variáveis biofísicas, por apresentar a maior quantidade de biomassa verde das plantas (Monteiro et al., 2012). 
A colheita foi realizada nas linhas centrais de cada parcela, em duas etapas: aos 92 e aos 102 dias após a semeadura (DAS). Assim, a área colhida correspondeu a $7 \mathrm{~m}^{2}$. A massa de grãos colhida foi corrigida para $13 \%$ de teor de umidade.

As medidas de reflectância foram obtidas com o espectrorradiômetro portátil Spectron SE-590, (Spectron Eng. Inc, Denver, Columbia-EUA) em 252 bandas, na faixa espectral de 350 a $1.100 \mathrm{~nm}$, com 2,7 nm de resolução espectral, e foram transformadas em fator de reflectância, conforme Steffen (1988). Em cada parcela, foram coletadas duas medidas de reflectância a 3,0 $\mathrm{m}$ de altura do dossel aproximadamente, com um campo de visada instantâneo (IFOV) de $15^{\circ}$, o que resultou em uma área de $0,49 \mathrm{~m}^{2}$. As medidas radiométricas foram realizadas no estádio R6 de desenvolvimento da cultura, em um período de duas horas, que incluiu o meio-dia solar, ou seja, entre 11:00 e 13:00 h (hora local), período em que as entrelinhas encontravam-se totalmente iluminadas, como tentativa de padronizar as condições de iluminação.

Os valores de reflectância das bandas espectrais dos sensores HRVIR, ETM+ e Modis foram simulados por meio dos dados hiperespectrais coletados pelo radiômetro de campo e, posteriormente, utilizados no cálculo dos índices de vegetação SR, NDVI, Savi e EVI: $\mathrm{SR}=\mathrm{IVP} / \mathrm{V}, \mathrm{NDVI}=[(\mathrm{IVP}-\mathrm{V}) /(\mathrm{IVP}+\mathrm{V})]$, Savi $=(1+\mathrm{L}) \times[(\mathrm{IVP}-\mathrm{V}) /(\mathrm{IVP}+\mathrm{V}+\mathrm{L})]$, $\mathrm{EVI}=\mathrm{G}[(\mathrm{IVP}-\mathrm{V}) /(\mathrm{IVP}+\mathrm{C} 1 \times \mathrm{V}-\mathrm{C} 2 \times \mathrm{A}+\mathrm{L})]$, em que: IVP (reflectância na região do infravermelho próximo), $\mathrm{V}$ (reflectância na região do vermelho) e A (reflectância na região do azul) são as bandas referentes aos sensores HRVIR, ETM+e Modis, simuladas com a média de reflectância das bandas estreitas que coincidem com as bandas do azul, vermelho e infravermelho próximo respectivas aos satélites Spot 4, Landsat 7 e Terra; L é uma constante de correção para o solo, considerada igual a 0,5 para Savi e 1 para EVI, mais adequada para diferentes tipos de solo (Huete, 1988); C1 é o coeficiente de correção dos efeitos atmosféricos para o vermelho $(6,0)$; C2 é o coeficiente de correção para os efeitos atmosféricos para o azul $(7,5)$; L é o fator de correção para a interferência do solo $(1,0)$; e G é o fator de ganho $(2,5)$ (Huete et al., 1997).

A partir dos dados de radiometria de campo, foi realizada a simulação das bandas individuais do $\mathrm{V}$ e IVP, para os diferentes sensores, conforme a função de resposta espectral normalizada de cada banda dos sensores (Miura et al., 2006). Para as bandas tratadas no presente trabalho, as funções de resposta normalizada dos sensores HRVIR, ETM+ e Modis/ Terra foram obtidas em National Aeronautics and Space Administration (2012), Astrium an Eads Company (2011) e National Aeronautics and Space Administration (2011), respectivamente. As bandas espectrais estão nas seguintes localizações para os sensores: HRVIR, V 0,61-0,68 mm e IVP 0,78-0,89 $\mathrm{mm}$; ETM+, V 0,63-0,69 mm e IVP 0,75-0,90 mm; Modis, A 0,46-0,48 mm, V 0,62-0,67 mm e IVP $0,84-0,88 \mathrm{~mm}$.

Os modelos de regressão, utilizados para estudar a relação entre os índices de vegetação e as variáveis biofísicas, foram o linear $(y=a+b x)$, o polinomial do segundo grau $\left(y=a+b x+c^{2}\right)$ e o geométrico $\left(\mathrm{y}=\mathrm{ax}{ }^{\mathrm{b}}\right)$, em que y é a variável dependente (IAF ou produtividade), e x é a variável independente, que pode ser as bandas individuais do V ou do IVP e os índices de vegetação SR, NDVI, Savi e EVI para os sensores HRVIR, ETM+e Modis.

A equação do tipo geométrica foi utilizada em razão da natureza assintótica que as relações IAF-índices de vegetação podem apresentar (Xavier \& Vettorazzi, 2004; Viña et al., 2011). O modelo cúbico não foi utilizado, pois, na análise preliminar dos dados, não se observou diminuição do NDVI com o aumento do IAF. A análise da melhor relação entre IAF e produtividade e os dados de sensoriamento remoto foi realizada por meio do coeficiente de determinação $\left(\mathrm{R}^{2}\right)$.

Para testar se uma determinada variável independente explicou melhor a variação da variável dependente, construíram-se intervalos de confiança para a diferença das médias de $\mathrm{R}^{2}$ das relações, com uso da técnica de "bootstrap" a 5\% de confiança. Para tanto, primeiramente observou-se qual o sensor e a variável independente que resultaram no maior valor de $\mathrm{R}^{2}\left(\mathrm{R}_{\text {ref }}^{2}\right)$ para a estimativa das variáveis biofísicas. Esta variável independente foi utilizada como referência para comparação com as demais variáveis independentes $\left(\mathrm{R}_{\text {outros }}^{2}\right)$. A partir das amostras coletadas em campo, calculou-se a diferença dos $\mathrm{R}^{2}(\hat{\theta})$ dada por $\hat{\theta}=\mathrm{R}_{\text {outros }}{ }^{2} \mathrm{R}_{\text {ref; }}^{2}$, em seguida, a partir das amostras originais, realizou-se uma nova amostragem, com repetição dos dados das variáveis dependentes e independentes da melhor estimativa e da que se comparou; para esta nova amostragem, foi calculada a estatística de 1, e armazenada a replicação "bootstrap" 
$\hat{\theta}^{* \mathrm{~b}}$; os procedimentos descritos em 2 a 3 foram repetidos dez mil (B) vezes, e a estatística $\hat{\theta}^{* b}$ foi ordenada em ordem crescente; por fim, calculou-se B $\cdot \alpha / 2$ e B · $(1-\alpha / 2)$ (Martinez \& Martinez, 2008). Para verificar se estes índices/sensores foram melhores para se estimar as variáveis biofísicas, eles foram utilizados como referência para, por meio de "bootstrap", compará-los com os demais índices de vegetação/ sensores. A comparação se deu por meio da construção dos intervalos de confiança (IC 95\%) para a diferença média da população dos $\mathrm{R}^{2}$ da referência e dos $\mathrm{R}^{2}$ dos outros índices de vegetação/sensores.

\section{Resultados e Discussão}

A produtividade de grãos variou de 0,785 a $5,249 \mathrm{Mg} \mathrm{ha}^{-1}$, e o IAF variou de 0,56 a $8,71 \mathrm{~m}^{2} \mathrm{~m}^{-2}$. Os valores de produtividade alcançados no presente trabalho foram superiores aos encontrados por Mantovani et al. (2012) de 2,946 $\mathrm{Mg} \mathrm{ha}^{-1}$. Resultados similares foram encontrados por Lima et al. (2010), em que o máximo valor de IAF encontrado foi próximo de $8 \mathrm{~m}^{2} \mathrm{~m}^{-2}$. A correlação entre a produtividade e o IAF foi de 0,85 .

As relações entre as variáveis biofísicas (IAF e produtividade) e a banda do V são caracterizadas pela absorção da radiação, no comprimento de onda do vermelho, pelos pigmentos fotossintetizantes presentes nas folhas, enquanto a relação entre as variáveis biofísicas e a banda do IVP é caracterizada pela alta reflectância da energia do infravermelho próximo, em razão do espalhamento interno da radiação na folha (Gates et al., 1965). A relação entre as variáveis biofísicas e a banda do $\mathrm{V}$ foi negativa (Figura 1); esta banda explica pelo menos $72 \%$ de suas variações (Tabela 1). Como a banda do $\mathrm{V}$ está diretamente associada à quantidade de energia absorvida pelos pigmentos das folhas, para realizar a fotossíntese, e também ao teor de clorofila nas folhas, quanto maior a quantidade de biomassa verde, maior será a absorção
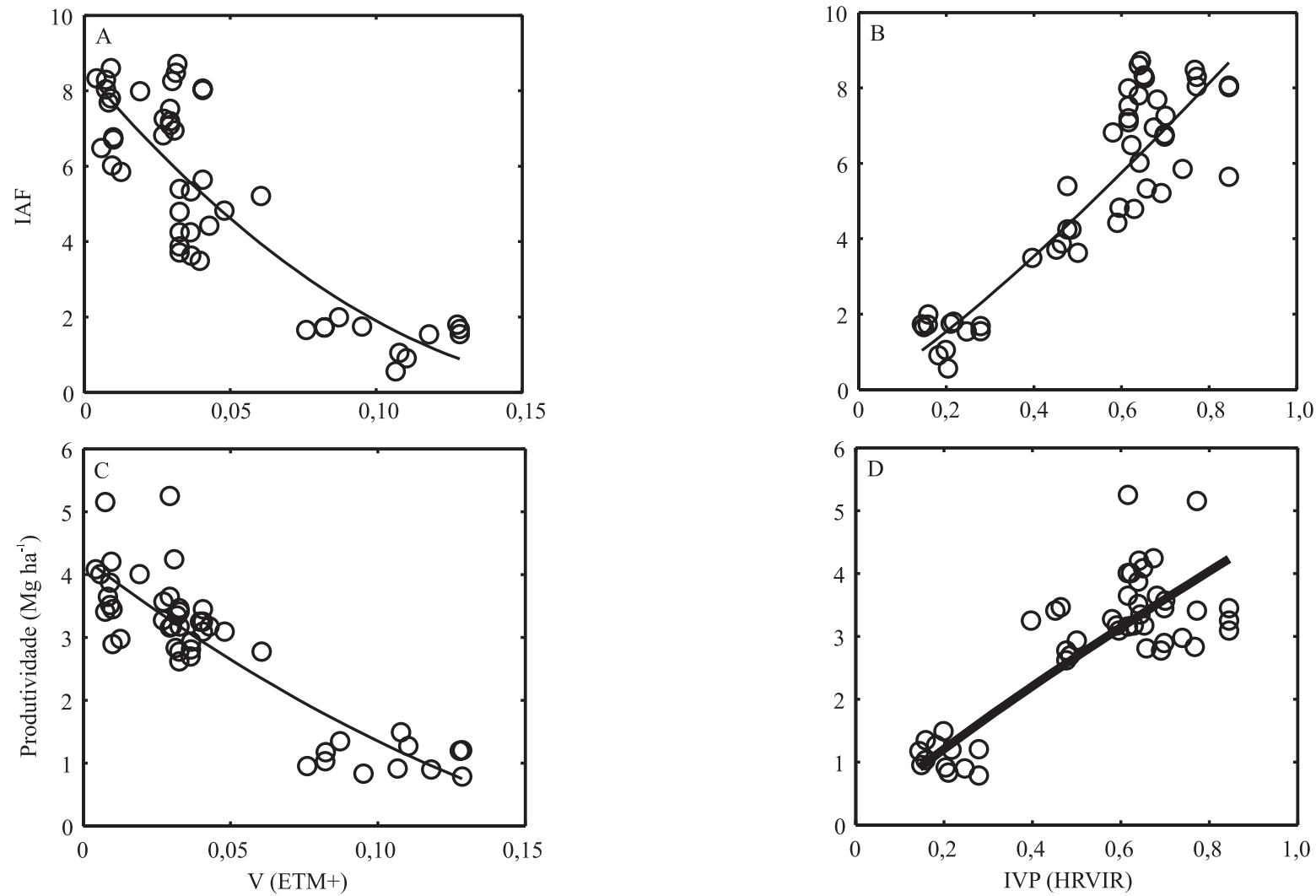

Figura 1. Diagramas de dispersão entre as variáveis biofísicas e as bandas do sensor Modis, vermelho (V) e infravermelho próximo (IVP), no estádio R6 da cultura do feijoeiro. A, IAF e V (ETM+); B, IAF e IVP (HRVIR); C, produtividade e V (ETM+); e D, produtividade e V (HRVIR). 
da radiação. As relações entre as variáveis biofísicas e o IVP foram positivas, em razão de haver um maior espalhamento da radiação pelas folhas nesta faixa espectral, ou seja, quanto maior o IAF, maior é o espalhamento. Para a relação IVP e variáveis biofísicas, o $\mathrm{R}^{2}$ foi sempre maior que 0,78 .

A banda do IVP apresentou melhores resultados na estimativa das variáveis biofísicas analisadas para os diferentes sensores (Tabela 1). Para a relação entre a banda $\mathrm{V}$, dos diferentes sensores, e as variáveis biofísicas, o modelo de regressão que melhor se ajustou aos dados foi o polinomial de segundo grau, enquanto para a banda do IVP foi o geométrico.

$\mathrm{Na}$ análise dos índices de vegetação, as relações para estimativa do IAF e da produtividade foram positivas (Figura 2), uma vez que estes respondem crescentemente à biomassa verde que, por sua vez, é associada positivamente à produtividade (Asrar et al., 1984). O EVI aparece como o melhor índice na estimativa do IAF (Tabela 2 e Figura 2), com $\mathrm{R}^{2}$ de 0,867 . Para os sensores HRVIR e ETM+, o índice Savi foi o que melhor estimou esta variável, com $\mathrm{R}^{2} \mathrm{de}$ 0,866 e 0,865 , respectivamente. Papadavid et al. (2013) estudaram a relação entre o IAF do feijão e o NDVI, que foi calculado com bandas referentes às do sensor $\mathrm{ETM}+$ simulado de dados de radiômetro, e obtiveram $\mathrm{R}^{2}$ superior a 0,88 , que é maior que os encontrados no presente trabalho.

$\mathrm{Na}$ estimativa da produtividade (Tabela $3 \mathrm{e}$ Figura 2), o NDVI, calculado com bandas do sensor Modis, foi o índice que melhor estimou esta variável com $\mathrm{R}^{2}=0,861$. Para esta variável, o modelo que melhor se ajustou aos índices de vegetação foi o geométrico. Monteiro et al. (2012) encontraram na relação do tipo linear entre índices de vegetação hiperspectrais e o IAF e a produtividade do feijão, $R^{2}$ de 0,87 e 0,84 , respectivamente, ou seja, próximos aos encontrados no presente trabalho com bandas largas.

Os maiores valores de $\mathrm{R}^{2}$ das relações entre o IAF e a produtividade e os índices de vegetação foram encontrados com o EVI/Modis e o NDVI/Modis, respectivamente. Quanto ao IC para o IAF, que teve como referência o EVI/Modis, quando as barras de erro da Figura 3 contém o zero (*) significa que não podemos afirmar que o EVI/Modis apresenta melhores relações que o IV/banda considerado para estimar a variável biofísica. Assim, o EVI/Modis só foi superior para estimar o IAF quando se utilizou apenas a banda V. O IVP, SR, Savi e o NDVI, calculados por meio dos sensores, têm a mesma eficiência para a estimativa do IAF que o EVI calculado pelas bandas do Modis.

As bandas do V e IVP, independentemente do sensor, e tendo-se como referência o Modis_NDVI, não foram tão eficientes para estimar a produtividade (Figura 4). No entanto, os índices de vegetação calculados pelos diferentes sensores (Modis_SR, HRVIR_SR, HRVIR_NDVI, HRVIR_Savi e ETM+_NDVI) foram tão eficientes para a estimativa da produtividade quanto o Modis_NDVI.

Com relação à avaliação das bandas espectrais da região do azul, vermelho e infravermelho de diferentes sensores, e de índices de vegetação

Tabela 1. Coeficiente de determinação $\left(\mathrm{R}^{2}\right)$, valor p e parâmetros $(\mathrm{a}, \mathrm{b}$ e c) dos modelos de regressão polinomial e geométrico, entre as bandas vermelho (V) e infravermelho próximo (IVP) dos índices de vegetação estudados com o índice de área foliar (IAF) e a produtividade, no estádio R6 da cultura do feijoeiro.

\begin{tabular}{|c|c|c|c|c|c|c|c|c|}
\hline Sensor & Variável & Modelo de regressão & Bandas & $\mathrm{a}$ & $\mathrm{b}$ & $\mathrm{c}$ & $\mathrm{R}^{2}$ & $\mathrm{p}$ \\
\hline \multirow{4}{*}{ Modis } & \multirow{2}{*}{ IAF } & polinomial & $\mathrm{V}$ & 8,82 & 92,16 & 229,4 & 0,721 & $<0,01$ \\
\hline & & geométrico & IVP & 10,48 & 1,20 & - & 0,835 & $<0,01$ \\
\hline & \multirow{2}{*}{ Produtividade } & polinomial & V & 4,39 & 36,41 & 58,7 & 0,766 & $<0,01$ \\
\hline & & geométrico & IVP & 4,85 & 0,87 & - & 0,787 & $<0,01$ \\
\hline \multirow{4}{*}{ HRVIR } & \multirow{2}{*}{ IAF } & polinomial & $\mathrm{V}$ & 8,79 & 91,74 & 231,3 & 0,722 & $<0,01$ \\
\hline & & geométrico & IVP & 10,62 & 1,20 & - & 0,836 & $<0,01$ \\
\hline & \multirow{2}{*}{ Produtividade } & polinomial & $\mathrm{V}$ & 4,38 & 36,39 & 61,3 & 0,768 & $<0,01$ \\
\hline & & geométrico & IVP & 4,90 & 0,87 & - & 0,788 & $<0,01$ \\
\hline \multirow{4}{*}{ ETM+ } & \multirow{2}{*}{ IAF } & polinomial & $\mathrm{V}$ & 8,55 & 90,71 & 242,2 & 0,735 & $<0,01$ \\
\hline & & geométrico & IVP & 10,71 & 1,20 & - & 0,835 & $<0,01$ \\
\hline & \multirow{2}{*}{ Produtividade } & polinomial & $\mathrm{V}$ & 4,27 & 35,71 & 64,8 & 0,775 & $<0,01$ \\
\hline & & geométrico & IVP & 4,93 & 0,87 & - & 0,787 & $<0,01$ \\
\hline
\end{tabular}



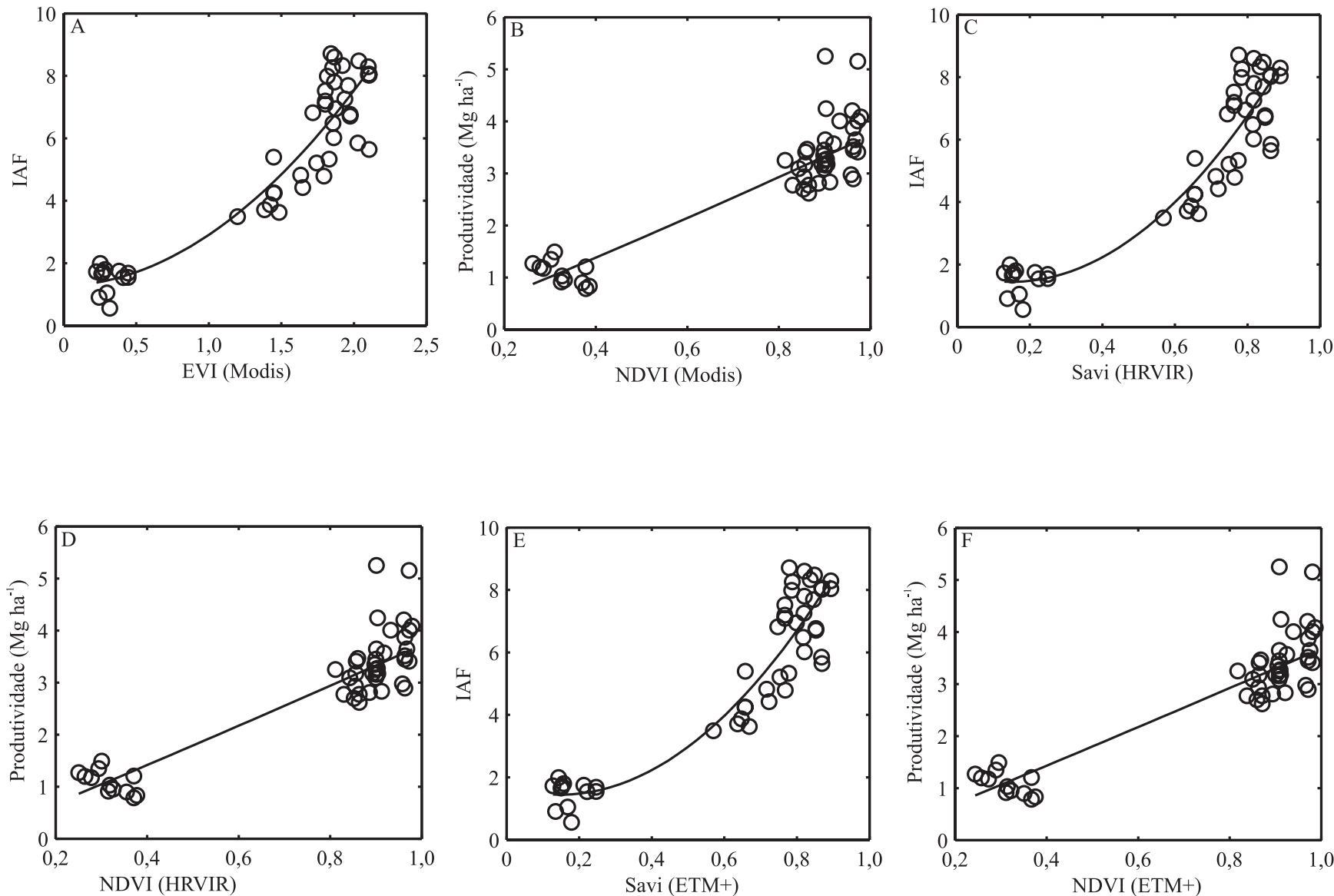

Figura 2. Diagramas de dispersão entre as variáveis biofísicas e os índices de vegetação mais bem ajustados dos sensores analisados no estádio R6 da cultura do feijoeiro. A, índice de área foliar (IAF) e EVI (Modis); B, produtividade e NDVI (Modis); C, IAF e Savi (HRVIR); D, produtividade e NDVI (HRVIR); E, IAF e Savi (ETM+); e F, produtividade e NDVI (ETM+).

Tabela 2. Coeficiente de determinação $\left(\mathrm{R}^{2}\right)$, valor $\mathrm{p}$ e parâmetros ( $\mathrm{a}, \mathrm{b}$ e c) do modelo de regressão geométrico e polinomial, entre os índices de vegetação e o índice de área foliar, no estádio R6 da cultura do feijoeiro.

\begin{tabular}{lccccccc}
\hline Sensor & Índice de & Modelo de & \multicolumn{3}{c}{ Parâmetro } & $\mathrm{R}^{2}$ & $\mathrm{p}$ \\
\cline { 4 - 6 } & vegetação & regressão & $\mathrm{a}$ & $\mathrm{b}$ & $\mathrm{c}$ & & \\
\hline \multirow{3}{*}{ Modis } & SR & Geométrico & 1,13 & 0,52 & - & 0,817 & $<0,01$ \\
& NDVI & Geométrico & 7,16 & 1,45 & - & 0,853 & $<0,01$ \\
& Savi & Polinomial & 1,74 & $-3,88$ & 12,6 & 0,865 & $<0,01$ \\
& EVI & Polinomial & 1,27 & 0,15 & 1,5 & 0,867 & $<0,01$ \\
\hline \multirow{3}{*}{ HRVIR } & SR & Geométrico & 1,14 & 0,52 & - & 0,817 & $<0,01$ \\
& NDVI & Geométrico & 7,14 & 1,41 & - & 0,853 & $<0,01$ \\
& Savi & Polinomial & 1,73 & $-3,77$ & 12,6 & 0,866 & $<0,01$ \\
\hline \multirow{3}{*}{ ETM+ } & SR & Geométrico & 1,23 & 0,47 & - & 0,794 & $<0,01$ \\
& NDVI & Geométrico & 7,03 & 1,37 & - & 0,852 & $<0,01$ \\
& Savi & Polinomial & 1,72 & $-3,68$ & 12,4 & 0,865 & $<0,01$ \\
\hline
\end{tabular}

Tabela 3. Coeficiente de determinação $\left(\mathrm{R}^{2}\right)$, valor $\mathrm{p}$ e parâmetros (a e b) do modelo de regressão geométrico, entre os índices de vegetação e a produtividade, no estádio R6 da cultura do feijoeiro.

\begin{tabular}{|c|c|c|c|c|c|}
\hline \multirow[t]{2}{*}{ Sensor } & \multirow{2}{*}{$\begin{array}{l}\text { Índice de } \\
\text { vegetação }\end{array}$} & \multicolumn{2}{|c|}{ Parâmetro } & \multirow[t]{2}{*}{$\mathrm{R}^{2}$} & \multirow[t]{2}{*}{$\mathrm{p}$} \\
\hline & & $\mathrm{a}$ & b & & \\
\hline \multirow{4}{*}{ Modis } & SR & 0,94 & 0,39 & 0,805 & $<0,01$ \\
\hline & NDVI & 3,73 & 1,09 & 0,861 & $<0,01$ \\
\hline & Savi & 4,04 & 0,75 & 0,844 & $<0,01$ \\
\hline & EVI & 2,32 & 0,63 & 0,850 & $<0,01$ \\
\hline \multirow{3}{*}{ HRVIR } & SR & 0,95 & 0,38 & 0,804 & $<0,01$ \\
\hline & NDVI & 3,72 & 1,06 & 0,859 & $<0,01$ \\
\hline & Savi & 4,05 & 0,74 & 0,844 & $<0,01$ \\
\hline \multirow{3}{*}{ ETM } & SR & 1,00 & 0,35 & 0,780 & $<0,01$ \\
\hline & NDVI & 3,68 & 1,03 & 0,858 & $<0,01$ \\
\hline & Savi & 4,02 & 0,73 & 0,844 & $<0,01$ \\
\hline
\end{tabular}


gerados por elas, na estimativa da produtividade e do IAF do feijoeiro, sabe-se que a resposta do sensor ao alvo também dependerá da resolução espacial e radiométrica do sensor. A resolução espacial dos sensores HRVIR, ETM+ e Modis são distintas, pois, na região do vermelho, as resoluções espaciais são, respectivamente, 20,30 e $250 \mathrm{~m}$. A resolução radiométrica do HRVIR e ETM+ é de
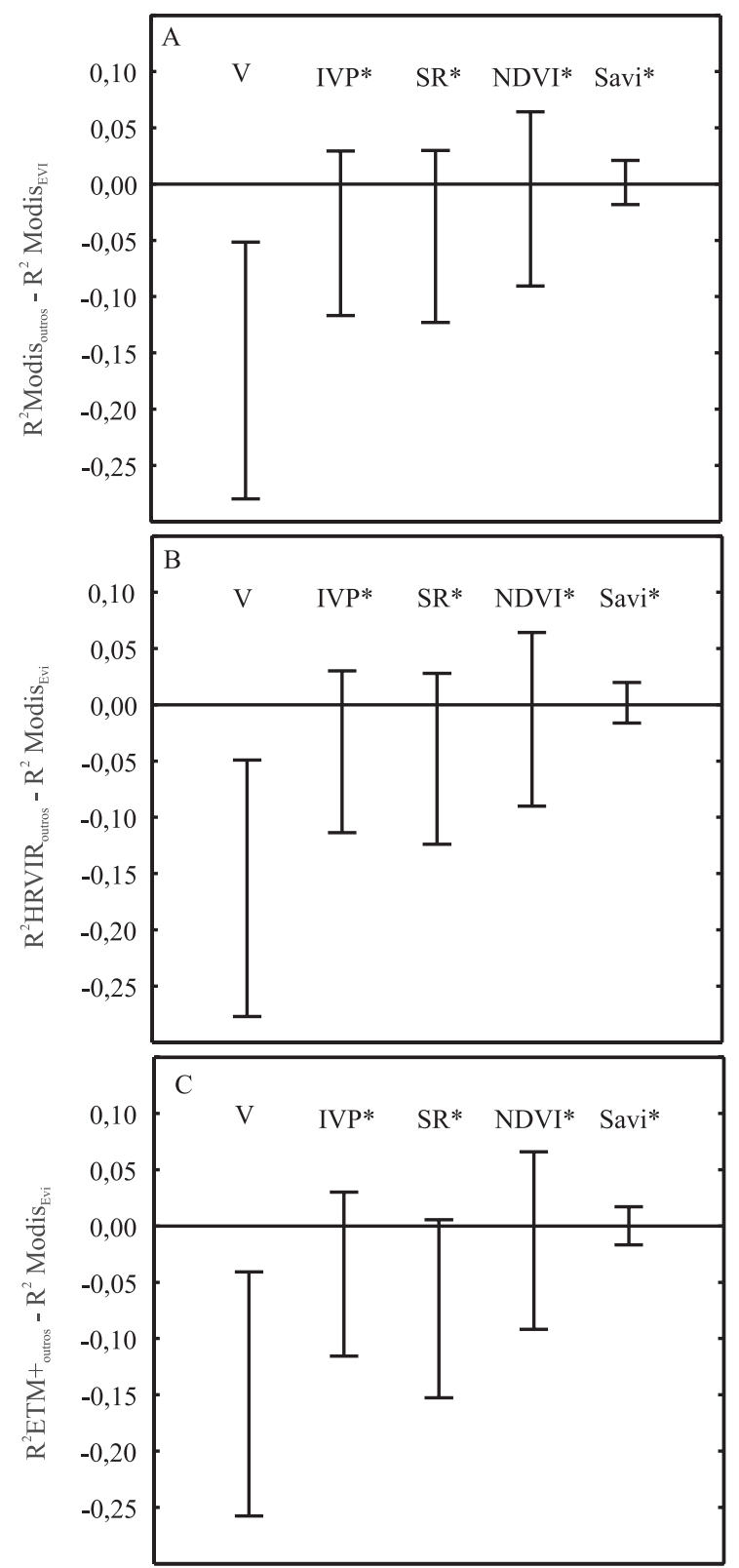

Figura 3. Intervalos de confiança (95\%) "bootstrap", para a diferença entre as médias de $\mathrm{R}^{2} \mathrm{EVI} /$ Modis e $\mathrm{R}^{2}$ dos outros sensores, e índices para estimativa do índice de área foliar. A, sensor Modis; B, sensor HRVIR; e C, sensor ETM+.
8 bits enquanto a do Modis é de 12 bits. Portanto, não se pode generalizar os resultados aqui obtidos. É importante que sejam utilizadas imagens destes sensores ou por meio de simulações destes em estudos posteriores. Podem-se, por exemplo, simular as resoluções de outros sensores (Miura et al., 2006) e avaliar seu desempenho pelas características
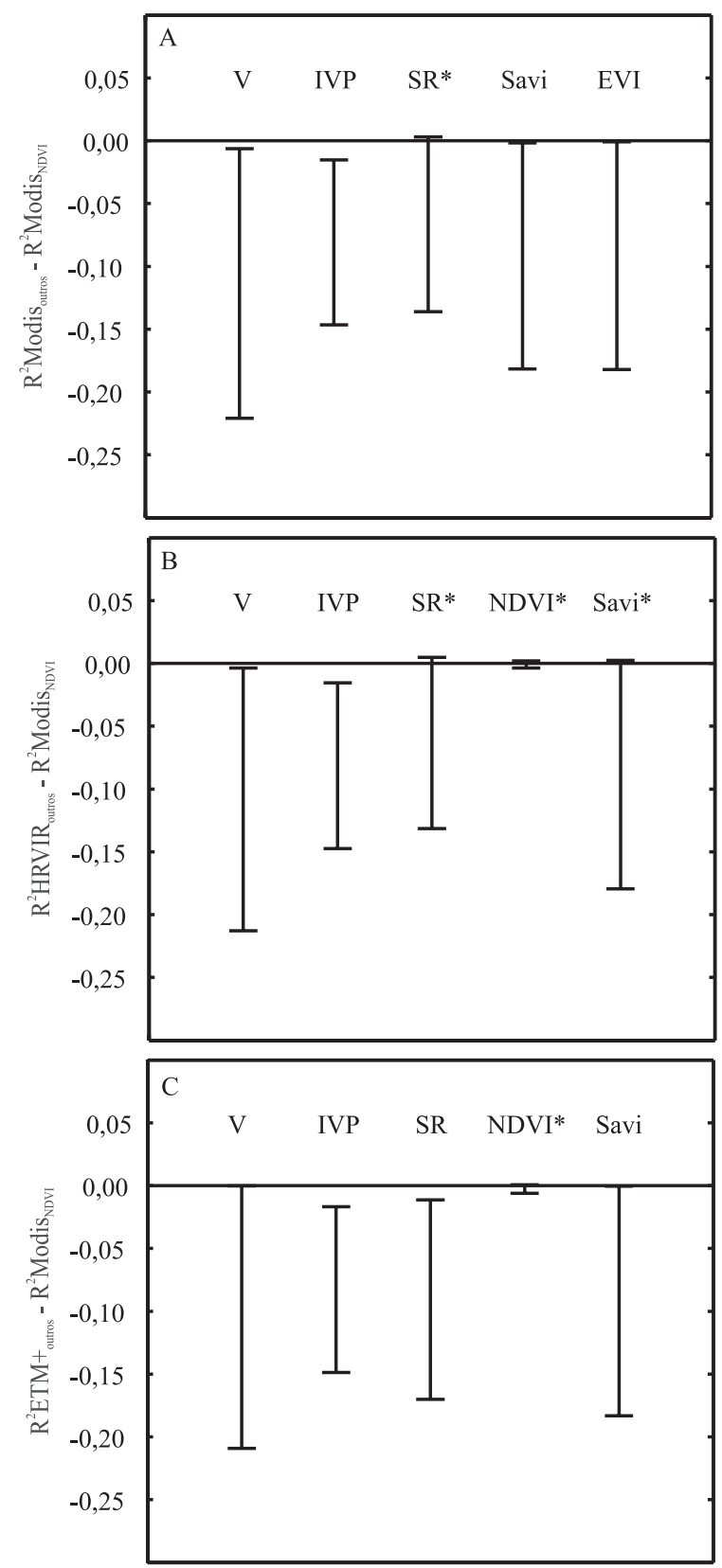

Figura 4. Intervalos de confiança por "bootstrap", para a diferença entre as médias de $\mathrm{R}^{2} \mathrm{EVI}_{\text {Modis }}$ e $\mathrm{R}^{2}$ de outros sensores, e índices para estimativa da produtividade. A, sensor Modis; B, sensor HRVIR; e C, sensor ETM+. 
biofísicas de culturas agrícolas, por meio de imagens do sensor Hyperion (National Aeronautics and Space Administration, 2007), que é um sensor hiperespectral, com 220 bandas a cada $10 \mathrm{~nm}$, na região de 400-2500 nm, resolução espacial (30 m) e radiométrica de 12 bits.

\section{Conclusões}

1. A banda do IVP e os índices de vegetação SR, Savi, NDVI e EVI, dos sensores Modis, ETM+ e HRVIR, apresentam o mesmo potencial na estimativa do IAF da cultura do feijoeiro.

2. Não há diferença de produtividade entre as estimativas, a partir dos índices de vegetação NDVI e SR calculados de dados Modis, SR, NDVI e Savi do HRVIR, e o NDVI do ETM+.

\section{Agradecimentos}

A Coordenação de Aperfeiçoamento de Pessoal de Nível Superior (Capes), à Fundação de Amparo à Pesquisa do Espírito Santo (Fapes) e ao Conselho Nacional de Desenvolvimento Científico e Tecnológico (CNPq), por concessão de bolsa.

\section{Referências}

ASRAR, G.; FUCHS, M.; KANEMASU, E.T.; HATFIELD, J.L. Estimating absorbed photosynthetic radiation and leaf area index from spectral reflectance in wheat. Agronomy Journal, v.76, p.300-306, 1984. DOI: 10.2134/agronj1984.00021962007600020 $029 \mathrm{x}$.

ASTRIUM AN EADS COMPANY. SPOT Images: resolution and spectral bands. 2011. Available at: <http://www.spotimage.com/ web/en/233-resolution-and-spectral-bands.php>. Accessed on: 10 jun. 2011.

DORAISWAMY, P.C.; HATFIELD, J.L.; JACKSON, T.J.; AKHMEDOV, B. PRUEGER, J.; STERN, A. Crop condition and yield simulations using Landsat and MODIS. Remote Sensing of Environment, v.92, p.548-559, 2004. DOI: 10.1016/j. rse.2004.05.017.

FOOD AND AGRICULTURE ORGANIZATION OF THE UNITED NATIONS. FaoStat. 2013. Available at: <http://faostat. fao.org/site/567/DesktopDefault.aspx?PageID=567\#ancor $>$. Accessed on: 10 Apr. 2013.

GALVÃO, L.S.; ROBERTS, D.A.; FORMAGGIO, A.R.; NUMATA, I.; BREUNIG, F.M. View angle effects on the discrimination of soybean varieties and on the relationships between vegetation indices and yield using off-nadir Hyperion data. Remote Sensing of Environment, v.113, p.846-856, 2009. DOI: 10.1016/j.rse.2008.12.010.
GATES, D.M.; KEEGAN, H.J.; SCHALETER, J.C.; WEINDER, V.R. Spectral properties of plants. Applied Optics, v.4, p.11-20, 1965. DOI: 10.1364/AO.4.000011.

GEPTS, P.; FERNANDÉZ, F. Etapas de desarrollo de la planta de frijol común (Phaseolus vulgaris L.). Cali: CIAT, 1982. 10p.

HANKS, R.J.; KELLER, J.; RASMUSSEN, V.P.; WILSON, G.D. Line source sprinkler for continuous variable irrigation-crop production studies. Soil Science Society of America Journal, v.40, p.426-429, 1976. DOI: 10.2136/sssaj1976.03615995004000 030033x.

HUETE, A.R. A soil-adjusted vegetation index (SAVI). Remote Sensing of Environment, v.25, p.295-309, 1988. DOI: 10.1016/0034-4257(88)90106-X.

HUETE, A.R.; LIU, H.Q.; BATCHILY, K.; VAN LEEUWEN, W. A comparison of vegetation indices global set of TM images for EOS-MODIS. Remote Sensing of Environment, v.59, p.440-451, 1997. DOI: 10.1016/S0034-4257(96)00112-5.

LIMA, C.L.R. de; REINERT, D.J.; REICHERT, J.M.; SUZUKI, L.E.A.S. Produtividade de culturas e resistência à penetração de Argissolo Vermelho sob diferentes manejos. Pesquisa Agropecuária Brasileira, v.45, p.89-98, 2010. DOI: 10.1590/S0100-204X2010000100012.

MARTINEZ, W.L.; MARTINEZ, A.R. Computational statistics handbook with MATLAB. New York: Chapman and Hall, 2008. $792 p$.

MIURA, T; HUETE, A.R.; YOSHIOKA H. An empirical investigation of cross-sensor relationships of NDVI and red/ near-infrared reflectance using EO-1 Hyperion data. Remote Sensing of Environment, v.100, p.223-236, 2006. DOI: 10.1016/j. rse.2005.10.010

MANTOVANI, E.C.; MONTES, D.R.P.; VIEIRA, G.H.S.; RAMOS, M.M.; SOARES, A.A. Estimativa de produtividade da cultura do feijão irrigado em Cristalina-GO, para diferentes lâminas de irrigação como função da uniformidade de aplicação. Engenharia Agrícola, v.32, p.110-120, 2012. DOI: 10.1590/ S0100-69162012000100012.

MONTEIRO, P.F.C.; ANGULO FILHO, R.; XAVIER, A.C.; MONTEIRO, R.O.C. Assessing biophysical variable parameters of bean crop with hyperspectral measurements. Scientia Agricola, v.69, p.87-94, 2012. DOI: 10.1590/S0103-90162012000200001.

NATIONALAERONAUTICS AND SPACE ADMINISTRATION. Landsat 7: science data users handbook. Available at: <http:// landsathandbook.gsfc.nasa.gov/inst_cal/prog_sect8 2.html>. Accessed on: 18 Jan. 2012.

NATIONAL AERONAUTICS AND SPACE ADMINISTRATION. MODIS: characterization support team. Available at: $<$ http://mcst. gsfc.nasa.gov/index.php? section=32>. Accessed on: 10 June 2011.

PAPADAVID, G.; FASOULA, D.; HADJIMITSIS, M.; PERDIKOU, P.S.; HADJIMITSIS, D.G. Image based remote sensing method for modeling black-eyed beans (Vigna unguiculata) leaf area index (LAI) and crop height $(\mathrm{CH})$ over cyprus. Central European Journal of Geosciences, v.5, p.1-11, 2013. DOI: 10.2478/s13533-012-0112-0. 
ROUSE JUNIOR, J.W.; HAAS, R.H.; DEERING, D.W.; SCHELL, J.A.; HARLAN, J.C. Monitoring the vernal advancement and retrogration (green wave effect) of natural vegetation. Greenbelt: NASA, 1974. 371p. (NASA/GSFC type III final report).

SANCHES, I.D.A.; EPIPHANIO, J.C.N.; FORMAGGIO, A.R. Culturas agrícolas em imagens multitemporais do satélite Landsat. Agricultura em São Paulo, v.52, p.83-96, 2005.

SANTOS, H.G. dos; JACOMINE, P.K.T.; ANJOS, L.H.C. dos; OLIVEIRA, V.A. de; OLIVEIRA, J.B. de; COELHO, M.R.; LUMBRERAS, J.F.; CUNHA, T.J.F. (Ed.). Sistema brasileiro de classificação de solos. 2.ed. Rio de Janeiro: Embrapa Solos, 2006. $306 \mathrm{p}$.

SORATTO, R.P.; CARVALHO, M.A.C. de; ARF, O. Teor de clorofila e produtividade do feijoeiro em razão da adubação nitrogenada. Pesquisa Agropecuária Brasileira, v.39, p.845-901, 2004. DOI: 10.1590/S0100-204X2004000900009.
STEFFEN, C.A. Spectron SE-590: manual de referência. São José dos Campos: Instituto Nacional de Pesquisas Espaciais, 1988. p.9-14.

VIÑA, A.; GITELSON, A.A.; NGUY-ROBERTSON, A.L.; PENG, Y. Comparison of different vegetation indices for the remote assessment of green leaf area index of crops. Remote Sensing of Environment, v.115, p.3468-3478, 2011. DOI: 10.1016/j. rse.2011.08.010.

XAVIER, A.C.; VETTORAZZI, C.A. Mapping leaf area index through spectral vegetation indices in a subtropical watershed. International Journal of Remote Sensing, v.25, p.1661-1672, 2004. DOI: $10.1080 / 01431160310001620803$.

XAVIER, A.C.; VETTORAZZI, C.A. Monitoring leaf area index at watershed level through NDVI from LANDSAT-7/ETM+data. Scientia Agricola, v.61, p.243-252, 2004. DOI: 10.1590/ S0103-90162004000300001.

Recebido em 19 de novembro de 2012 e aprovado em 2 de abril de 2013 